glyoxaline-4 : 5-dicarboxyamide, which may yield either 7- or 9-methylxanthine (or, less likely, both) is being examined. In a different connexion, the reaction has been applied to quinoxaline-2 : 3-dicarboxyamide (III) to give alloxazine (IV) in 60 per cent yield.

R. A. Baxter.

University, Manchester.

F. S. SPRING.

${ }^{1}$ Rec. trav. Chim., 10, 4 (1891).

'Weidel and Roitner, Monatsh., 17, 174 (1896).

${ }^{3}$ Rinkes, Rec. trav. Chim., 46, 268 (1927).

${ }^{4}$ Gabriel and Sonn, Ber., 40, 4857 (1907). Cf. Kuhling, Ber., 28 1968 (1895). Kuhn and Cook, Ber., 70, 761 (1937).

\section{Loss of Inorganic Constituents on Combustion of Coal and Coke}

THe standard method for preparing coal and coke ash for analysis is to burn off the carbonaceous matter by placing the sample in a silica tray contained in a muffle furnace heated to $800^{\circ} \mathrm{C}$. While this procedure clearly leads to the volatilization of a proportion of the sulphur, arsenic and other volatile elements, it has hitherto been considered a valid assumption that the whole of the sodium, potassium, calcium, magnesium, silica, iron, alumina and phosphorus contained in the fuel is also present in the ash.

Observations made in the course of an investiga. tion relating to flue solids have led us to suspect the correctness of this assumption.

To decide the issue, coal and coke were fired in a bomb such as is used for calorific svalue determinations or heats of combustion. By this method all the inorganic components of a fuel are retained in the bomb. Preliminary experiments indicate that, in the case of sodium or potassium, 0-60 per cent of the original content expressed as percentage of ash may be lost when the ash of a fuel is prepared by combustion in a muffle furnace kept at $800^{\circ} \mathrm{C}$. with free access of air. The bomb method of combustion for the concentration of the inorganic constituents of a fuel is a valuable technique which may reveal the presence of unsuspected elements, as well as the fact that certain elements are present in greater proportion than is usually thought. It may also reveal that determinations of elements (for example, phosphorus) by a method which depends upon their concentration in the ash by combustion of the carbonaceous material of a fuel are in error.

A. H. Edwards. A. O. Pearce.

British Coal Utilisation Research Association,

Coombe Springs, Kingston-on-Thames. July 28.

\section{Metabolism of Gill Epithelium of a Freshwater Mussel}

GRAY compared (1928) the chemical mechanism underlying rhythmic ciliary movement in mussel gills with that of muscle, especially the frog's heart, and found an essential similarity. Our knowledge of muscle chemistry, especially as regards the carbohydrate catabolism, has since increased considerably, and it appears worth while now to attempt a renewed comparison.

I have studied the epithelium of the gills of the freshwater mussel Dreissensia, which will retain its activity and oxygen consumption unaltered for at least 24 hours in ordinary fresh water after being cut off. When the gills are shaken in the Warburg apparatus, many cells will drop off, but even these will remain active.

As a basis for comparison, the nitrogen content has proved more suitable than either wet or dry weight. 13 per cent of the nitrogen found is located in the gill skeleton, the rest in the epithelium, and the gills on the two sides of the animal correspond within 3 per cent. The oxygen consumption per mgm. N/hour at $20^{\circ} \mathrm{C}$. is $18.7 \mu l$. and the R.Q. $0 \cdot 87$ with a standard deviation of 0.04 .

Ciliary movements are inhibited in about an hour by 25 millinormal sodium fluoride and in 45 minutes by $3 \cdot 3$ millinormal monoiodoacetamide, but not at all by phloridzin in concentrated solution. All these determinations were made at $20^{\circ}$ and $p \mathrm{H} \quad 7 \cdot 5-7 \cdot 6$. Complete lack of oxygen stops the cilia in $30 \mathrm{~min}$. at $p \mathbf{H} 6 \cdot 5$, but the effect at $p \mathbf{H} 8 \cdot 5$ is slight only. This agrees with a finding by Clark and Eggleton (1938) that the anaerobic formation of lactic acid in the frog's heart is inhibited at acid reactions.

Tests were made to find out whether lactic acid was formed in the ciliary epithelium under anaerobic conditions at $p \mathrm{H} 8 \cdot 5$, but while a considerable amount of acid. was formed, as shown by the liberation of carbon dioxide in the Warburg vessels, the special test for lactic acid (Koenemann, ]940) showed no increase whatever.

An attempt was made to isolate the acid by extraction with ether after precipitation of proteins. It turned out to be volatile and to smell like lower fatty acids, but sufficient material for chemical identification was not obtained.

When the ethereal solution was shaken with normal caustic soda and this afterwards titrated electrometrically with $0 \cdot 1 \mathrm{~N}$ hydrochloric acid, the titration curve showed, a definite inflexion at $p \mathrm{H} 5$, again indicating that the substance differs from lactic acid which shows a $p \mathrm{~K}$ of $3 \cdot 9$.

Lower fatty acids are produced by anaerobiosis in certain animals, notably valerianic acid in Ascaris (Weinland, 1901), and several formulæ have been proposed to account for their formation from sugar. These require the simultaneous formation of carbon dioxide, which was observed also in the case of Ascaris, but corresponding tests on Dreissensia gills gave a negative result. No carbon dioxide was produced anaerobically from half the gills of four animals in 5-7 hours as compared with the other half, determined at the beginning of the period.

On the other hand, it was shown by similar comparisons that glyeogen, found to make up about $\mathbf{1 . 5}$ per cent of the fresh weight, disappears from the gills both in aerobic and in anaerobic metabolism, but at about three times the rate during anaerobiosis. The glycogen disappearing is only partly catabolized, as reducing substances are found in the medium at the end of the experimental periods.

The respiratory quotient of 0.87 shows that some other substance than carbohydrate must be regularly catabolized, and a definite formation of ammonium proportional to the oxygen consumption was observed.

The experiments are to be continued, but so far as they go they are sufficient to show that the metabolic mechanism supplying energy for the ciliary movements in Dreissensia differs definitely from that of muscular contraction in vertebrates.

Wenner-Gren Institute Christer Wernstedt. of Experimental Biology,

Stockholm. July 30 . 